\title{
Remote sensing of terrestrial chlorophyll fluorescence from space
}

Christian Frankenberg, Joseph Berry, Luis Guanter, and Joanna Joiner

High-resolution spectrometers enable new avenues in global carbon cycle research, including the first accurate retrievals of chlorophyll fluorescence from space as an indicator of photosynthetic activity.

During photosynthesis, part of the solar radiation absorbed by chlorophyll is re-emitted at longer wavelengths (fluorescence). Using new, high-resolution spectrometers, we can now measure this chlorophyll fluorescence from space, which can, in turn, be used to quantify photosynthetic activity and efficiency globally. Such measurements are important to reduce uncertainties in the global carbon cycle. Indeed, our ability to control the Earth's carbon budget in a warming climate depends critically on knowing where, when, and how $\mathrm{CO}_{2}$ is exchanged between the land and atmosphere. Gross primary production (GPP), the gross uptake of atmospheric $\mathrm{CO}_{2}$ through photosynthesis, constitutes the largest flux component in the global carbon budget. However, considerable uncertainties remain in GPP estimates and its seasonality.

Chlorophyll fluorescence has been used in laboratory-scale studies of photosynthesis for several decades. ${ }^{1}$ Chlorophyll molecules in plant leaves are organized into so-called photosynthetic units, macromolecular complexes that contain many chlorophyll molecules and 'reaction centers' that process the absorbed energy (see Figure 1 . In these centers, the pigment molecules act as 'antennas' to capture solar photons. A phenomenon known as quantum coherence delocalizes the absorbed photon (exciton) among all the chlorophyll molecules ${ }^{2}$ in the unit. The exciton can have one of several fates occurring at different rates. Specifically, the exciton can be used by the enzyme photosystem II (PS2) to take an electron from water to make oxygen, it can undergo radiationless decay, it can be re-emitted as a fluorescent photon, or it can be quenched by regulated non-photochemical trapping centers (NPQs). When the potential flux of electrons

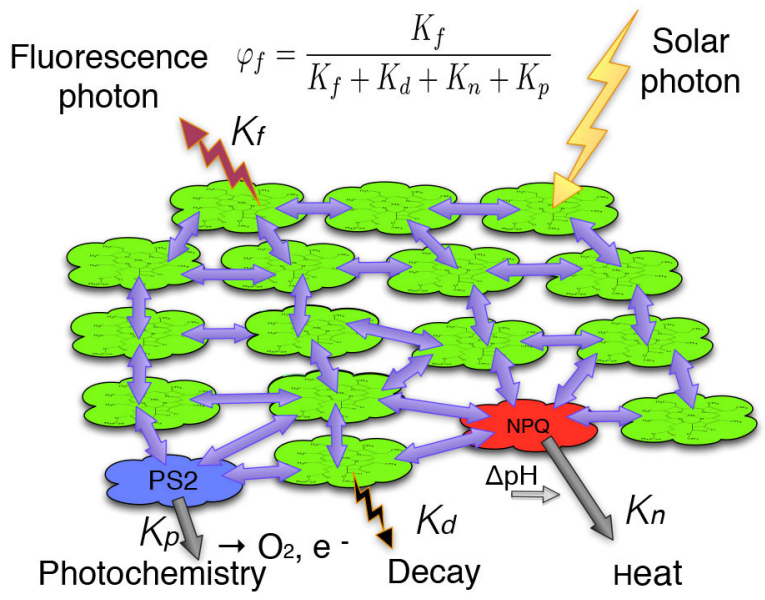

Figure 1. Arrays of chlorophyll molecules bound to protein absorb and process solar photons in plant leaves. A variable fraction $\left(\varphi_{f}\right.$, typically $\sim 1 \%$ ) of these leak back out as fluorescent photons. The absorbed photon can be lost as radiationless decay $\left(K_{d}\right)$, re-emitted as a fluorescent photon $\left(K_{f}\right)$, quenched by NPQs $\left(K_{n}\right)$, or used for photochemistry $\left(K_{p}\right)$. Photochemistry involves photosystem II (PS2), a photon-capturing enzyme. $e^{-}$: Electron. NPQs: Non-photochemical trapping centers.

exceeds the capacity to use them for making sugars, NPQ activity increases, resulting in diagnostic changes in fluorescence yield $\left(\varphi_{f}\right)$ while balancing supply with demand.

The fluorescence yield is determined by the ratio of its rate constant to the sum of all the rate constants for quenching of the exciton by these processes (see Figure 1). Solar-induced chlorophyll fluorescence (SIF) measured by satellites, therefore, represents events occurring at the molecular scale in the vegetation below and is directly proportional to the product of absorbed photosynthetic radiation and $\varphi_{f}$. When plants run 
short of water or temperatures are unfavorable, photosynthesis is slowed but light absorption continues. To rebalance, plants increase quenching by NPQs, resulting in a decrease in fluorescence through reductions in $\varphi_{f}$. Additionally, plants often decrease their leaf area when stressed, which decreases both photosynthesis and fluorescence further through reductions in absorbed radiation.

Typically, $\sim 1 \%$ of the solar energy captured by plants is reemitted by chlorophyll as fluorescence. This provides a distinct 'glow' at wavelengths of $\sim 690-800 \mathrm{~nm}$ that is quite specific to green plants. As this signal is very weak compared with the reflected solar radiation, the quantification of SIF from space has so far not been feasible, and vegetation remote sensing has solely relied on spectral indices. However, new spectrometers with high spectral resolution $(\sim 0.02-0.05 \mathrm{~nm}$ full-width half-maximum) in the range centered around 760nm now enable accurate and global fluorescence retrieval by measuring the depth of Fraunhofer lines, which are narrow absorption lines in the solar spectrum caused by chemical elements (such as iron and magnesium) in the sun's photosphere. An emission source at the Earth's surface-such as chlorophyll fluorescence-reduces the fractional depth of these lines, and
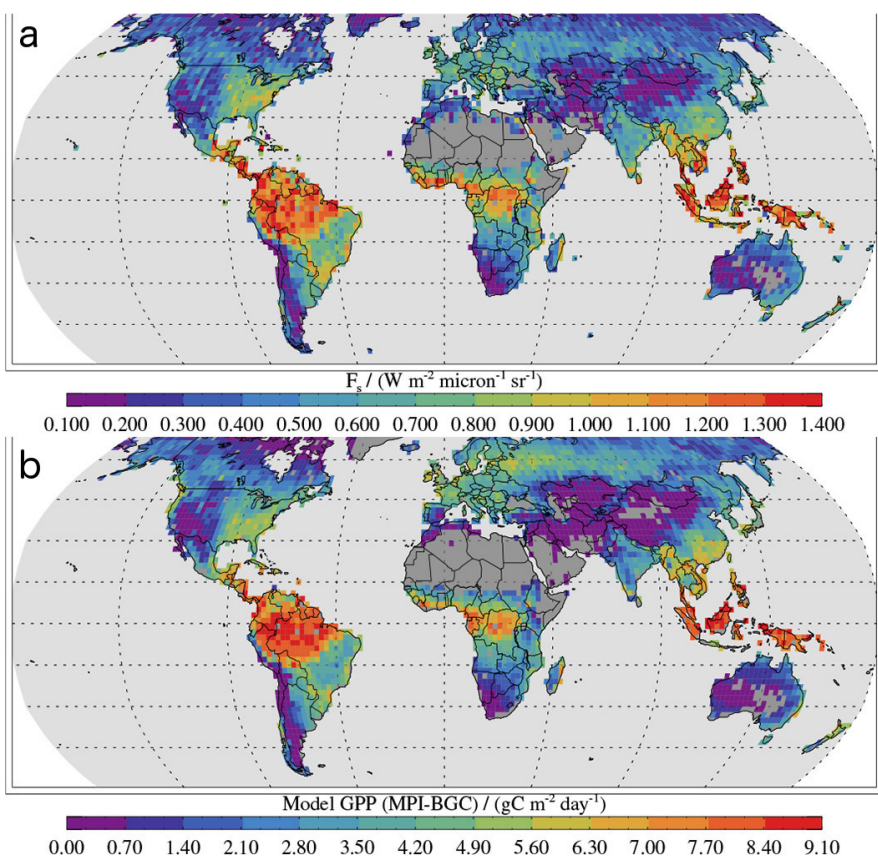

Figure 2. (a) Solar-induced chlorophyll fluorescence (SIF) as retrieved from GOSAT from June 2009 to December 2011. (b) Modeled gross primary production (GPP) for the same time period and locations. ${ }^{3,4}$ GOSAT: Global Greenhouse Gas Observation by Satellite. sr: Steradian. MPI-BGC: Max Planck Institute for Biogeochemistry.

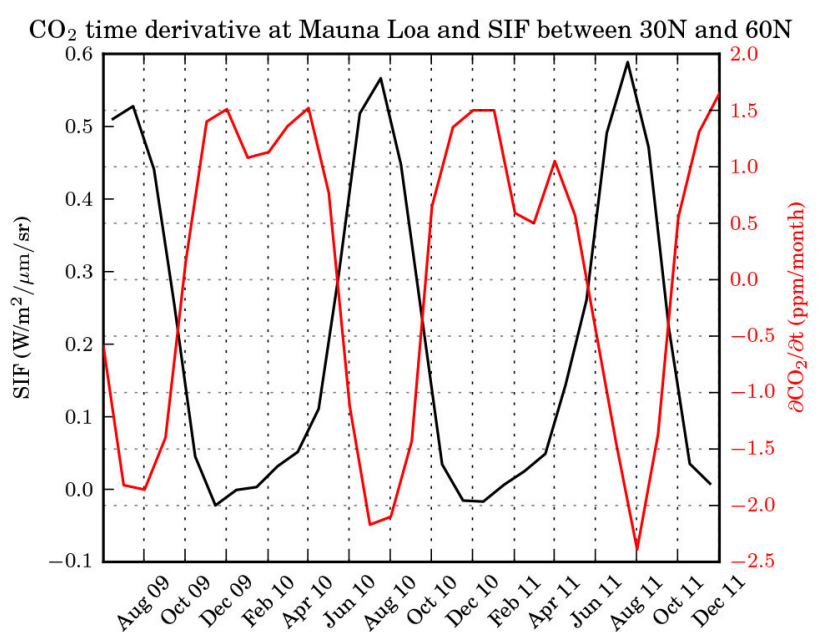

Figure 3. Time series of SIF (black line) averaged over the northern hemisphere $\left(30-60^{\circ} \mathrm{N}\right)$ and time derivatives of the atmospheric $\mathrm{CO}_{2}$ concentrations (red line) measured at Mauna Loa, Hawaii. ${ }^{11}$

they can be measured by the spectrophotometer. If, for instance, a Fraunhofer line is perfectly opaque ( $0 \%$ transmission), the radiance measured by a satellite simply comprises fluorescence from chlorophyll because it is essentially the only light source in this narrow wavelength window. In reality, Fraunhofer lines are not entirely opaque, and spectral fitting techniques ${ }^{5-7}$ are needed to disentangle the amount of fluorescence from solar light reflected by the Earth's surface and atmosphere.

For the first global retrievals of SIF, ${ }^{6-9}$ we measured part of the fluorescence emission spectrum $(\sim 755-775 \mathrm{~nm})$ using the Fourier transform spectrometer on board the Japanese GOSAT (Global Greenhouse Gas Observation by Satellite) satellite. ${ }^{10}$ The satellite was launched on 23 January 2009 into a Sun-synchronous orbit with an overpass time of 13:00 and was designed to determine greenhouse gas amounts $\left(\mathrm{CO}_{2}\right.$ and methane) in the atmosphere. Essentially, we measure the fractional depths of the Fraunhofer lines to derive SIF.

Figure 2 shows a global average of SIF as well as a corresponding map of modeled GPP, revealing strong correlations between both parameters. ${ }^{8}$ These observations are directly linked to photosynthesis, provide new information not available from greenness indices, and enable an entirely new perspective on the global carbon cycle. Observations of SIF can, therefore, have an important role in carbon cycle research and alleviate the large uncertainties in GPP measurements.

Figure 3 shows an example of how SIF is linked to the global carbon cycle and atmospheric $\mathrm{CO}_{2}$ abundance as measured 
at Mauna Loa, Hawaii. ${ }^{11}$ In boreal summer, vegetation in the northern hemisphere is most active, as indicated by maxima in SIF and high $\mathrm{CO}_{2}$ uptake (negative time derivative). In boreal winter, photosynthesis of most northern plants is inhibited (near-zero SIF), and respiration leads to substantial release of $\mathrm{CO}_{2}$ into the atmosphere. Thus, the SIF-to- $\mathrm{CO}_{2}$ flux relationship is inversely related (see Figure 3).

In summary, we have developed a technique to derive SIF from space measurements and showed empirically that these values are well-correlated with GPP. Although this is a big step in carbon cycle research, more work needs to be done to unravel the full potential of space-based SIF measurements. For example, plant physiological and radiative transfer modeling work is needed to consolidate the interpretation of fluorescence and its regulation from the canopy to the satellite footprint scale. The satellites currently in orbit were not specifically designed to quantify fluorescence and are, therefore, not optimal in terms of retrieval and temporal precision, and-most importantly-spatial sampling. However, the proposed FLuorescence EXplorer (FLEX) is a dedicated fluorescence mission by the European Space Agency. Moreover, the upcoming (in 2014) NASA Orbiting Carbon Observatory (OCO-2) mission will provide 100 times more data than GOSAT and will, therefore, greatly reduce retrieval uncertainties.

The authors are grateful for the Japanese GOSAT teams from the Japan Aerospace Exploration Agency, and the Japanese National Institute for Environmental Studies and Ministry of the Environment. The authors acknowledge ideas and advice from the participants in the New Methods to Measure Photosynthesis from Space workshop organized by the W. M. Keck Institute for Space Studies, California Institute of Technology. ${ }^{12}$ Part of the research described in this paper was carried out by the Jet Propulsion Laboratory, California Institute of Technology, under a contract with NASA. We thank Martin Jung and Markus Reichstein for providing the Max Planck Institute for Biogeochemistry (Jena, Germany) data set.

\section{Author Information}

\section{Christian Frankenberg}

Jet Propulsion Laboratory,

California Institute of Technology

Pasadena, CA

Christian Frankenberg is researcher whose work deals with remote sensing of atmospheric greenhouse gases, an interdisciplinary approach to surface and atmospheric remote sensing, and the global carbon cycle in general.

\section{Joseph Berry}

Department of Global Ecology

Carnegie Institution of Washington

Stanford, CA

Joe Berry is a staff scientist whose research has dealt with many aspects of photosynthesis. He has developed models that are widely used for simulating photosynthesis, stomatal conductance, and fluorescence of plants at scales from the leaf to the planet.

\section{Luis Guanter}

Institute for Space Sciences

Free University

Berlin, Germany

Luis Guanter leads a junior research group at the Free University that focuses on the retrieval and analysis of solar-induced chlorophyll fluorescence. His research interests are in the retrieval of land and atmospheric parameters from remote sensing systems.

\section{Joanna Joiner \\ NASA Goddard Space Flight Center \\ Greenbelt, MD}

Joanna Joiner has worked as an atmospheric physicist at the NASA Goddard Space Flight Center for over 15 years. In addition to conducting scientific research, she is the deputy project scientist for the NASA Aura satellite mission and acting US science team leader for the Ozone Monitoring Instrument onboard Aura.

\section{References}

1. G. Krause and E. Weis, Chlorophyll fluorescence and photosynthesis-the basics, Annu. Rev. Plant. Phys. 42, pp. 313-349, 1991.

2. G. S. Engel, T. R. Calhoun, E. L. Read, T.-K. Ahn, T. Mančal, Y.-C. Cheng, R. E. Blankenship, and G. R. Fleming, Evidence for wavelike energy transfer through quantum coherence in photosynthetic systems, Nature 446 (7137), pp. 782-786, 2007.

3. C. Beer, M. Reichstein, E. Tomelleri, P. Ciais, M. Jung, N. Carvalhais, C. Rodenbeck, M. Arain, D. Baldocchi, and G. Bonan, Terrestrial gross carbon dioxide uptake: global distribution and covariation with climate, Science 329 (5993), p. 834, 2010.

4. M. Jung, M. Reichstein, H. A. Margolis, A. Cescatti, A. D. Richardson, M. A. Arain, A. Arneth, et al., Global patterns of land-atmosphere fluxes of carbon dioxide, latent heat, and sensible heat derived from eddy covariance, satellite, and meteorological observations, J. Geophys. Res. 116, p. G00J07, 2011.

5. C. Frankenberg, A. Butz, and G. C. Toon, Disentangling chlorophyll fluorescence from atmospheric scattering effects in O-2 A-band spectra of reflected sun-light, Geophys. Res. Lett. 38, p. L03801, 2011. doi:10.1029/2010GL045896

6. J. Joiner, Y. Yoshida, A. P. Vasilkov, Y. Yoshida, L. A. Corp, and E. M. Middleton, First observations of global and seasonal terrestrial chlorophyll fluorescence from space, Biogeosciences 8 (3), pp. 637-651, 2011. doi:10.5194/bg-8-637-2011 
7. L. Guanter, C. Frankenberg, A. Dudhia, P. E. Lewis, J. Gómez-Dans, A. Kuze, H. Suto, and R. G. Grainger, Retrieval and global assessment of terrestrial chlorophyll fluorescence from GOSAT space measurements, Remote Sens. Env. 121, pp. 236-251, 2012. doi:10.1016/j.rse.2012.02.006

8. C. Frankenberg, J. Fisher, J. Worden, G. Badgley, S. Saatchi, J.-E. Lee, G. Toon, et al., New global observations of the terrestrial carbon cycle from GOSAT: patterns of plant fluorescence with gross primary productivity, Geophys. Res. Lett. 38 (17), p. L17706, 2011. doi:10.1029/2011GL048738

9. J. Joiner, Y. Yoshida, A. P. Vasilkov, E. M. Middleton, P. K. E. Campbell, Y. Yoshida, A. Kuze, and L. A. Corp, Filling-in of near-infrared solar lines by terrestrial fluorescence and other geophysical effects: simulations and space-based observations from SCIAMACHY and GOSAT, Atmos. Meas. Tech. 5 (4), pp. 809-829, 2012. doi:10.5194/amt-5-809-2012

10. T. Hamazaki, Y. Kaneko, A. Kuze, and K. Kondo, Fourier transform spectrometer for greenhouse gases observing satellite (GOSAT), Proc. SPIE 5659, p. 73, 2005. doi: $10.1117 / 12.581198$

11. GLOBALVIEW-CO2, Cooperative Atmospheric Data Integration Project-Carbon Dioxide. CD-ROM, National Oceanic and Atmospheric Administration Earth System Research Laboratory, Boulder, CO (also available on the Internet via Anonymous FTP to ftp.cmdl.noaa.gov, path: ccg/co2/GLOBALVIEW), 2011.

12. http://www.kiss.caltech.edu/workshops/photosynthesis2012/ New Methods to Measure Photosynthesis from Space workshop, California Institute of Technology. Accessed 8 February 2013. 